\title{
DISTOCIA MATERNA POR INÉRCIA UTERINA PRIMÁRIA ASSOCIADA AO CHOQUE HIPOGLICÊMICO EM CADELA: RELATO DE CASO
}

\author{
Fernanda Vieira Henrique ${ }^{1}$ \\ Angélica Oliveira da Silva ${ }^{2}$ \\ Amanda Valéria Angelo da Silva ${ }^{3}$ \\ Rosileide dos Santos Carneiro ${ }^{4}$ \\ Norma Lúcia de Sousa Araújo 5
}

HENRIQUE, F. V.; SILVA, A. O. da; SILVA, A. V. A. da; CARNEIRO, R. dos S.; ARAÚJO, N. de S. Distocia materna por inércia uterina primária associada ao choque hipoglicêmico em cadela: relato de caso. Arq. Ciênc. Vet. Zool. UNIPAR, Umuarama, v. 18, n. 3, p. 179-183, jul./set. 2015.

RESUMO: Distocia é a incapacidade de expulsão do feto por meio do canal do parto durante a parição. Relata-se um caso de distocia por inércia uterina primária associada o choque hipoglicêmico em uma cadela, SRD, de cinco anos de idade. O animal deu entrada no hospital veterinário, apresentando contrações abdominais e secreção vaginal de coloração esverdeada, hipotermia, obnubilação e hipoglicemia, caracterizando choque hipoglicêmico. Foi realizado tratamento emergencial e reversão da hipoglicemia. Foi realizada radiografia para verificação de fetos remanescentes e avaliação da estática fetal. A bioquímica sérica revelou hipocalcemia moderada e foi realizado tratamento com gluconato de cálcio e ocitocina, havendo a parição dos fetos. A distocia na clínica de cães e gatos é considerada de emergência. A estabilização do animal é de extrema importância. A realização de exames laboratoriais deve ser preconizada para avaliar a ocorrência de distúrbios eletrolíticos, promovendo um adequado tratamento e melhorando o prognóstico da paciente.

PALAVRAS-CHAVE: Parto distócico. Hipocalcemia. Hipoglicemia.

\section{MATERNAL DYSTOCIA BY PRIMARY UTERINE INERTIA ASSOCIATED WITH HYPOGLYCEMIC SHOCK IN DOG: CASE REPORT}

\begin{abstract}
Dystocia is the inability to expel the fetus through the birth canal during parturition. This is a report of a case of dystocia by primary uterine inertia associated with hypoglycemic shock in a five-year-old mongrel bitch. The animal was admitted to the veterinary hospital with abdominal contractions and greenish vaginal secretion, hypothermia, hypoglycemia and numbness, characterized as hypoglycemic shock. Emergency treatment and reversal of hypoglycemia were performed, as well as radiography to check for remaining fetuses and fetal static evaluation. Serum biochemistry revealed mild hypocalcemia and treatment was conducted with calcium gluconate and oxytocin, upon the birth of the fetuses. Clinic dystocia in dogs and cats is considered an emergency. The stabilization of the animal is of utmost importance. Laboratory tests should be recommended to assess the occurrence of electrolyte disturbances, promoting adequate treatment and improving the prognosis of the patient.
\end{abstract}

KEYWORDS: Dystocia. Hypocalcemia. Hypoglycemia.

\section{DISTOCIA MATERNA POR INERCIA UTERINA PRIMARIA ASOCIADA A CHOQUE HIPOGLUCÉMICO EN PERRA: RELATO DE CASO}

RESUMEN: Distocia es la incapacidad de expulsar el feto a través del canal durante el parto. Se presenta un caso de distocia por inercia uterina primaria asociada con el choque hipoglucémico en una perra, SRD, de cinco años de edad. El animal fue ingresado en el hospital veterinario, con contracciones abdominales y secreción vaginal de color verdoso, hipotermia, hipoglucemia y entumecimiento, caracterizando choque hipoglucémico. Se realizó un tratamiento de emergencia y reversión de la hipoglucemia. Se realizó radiografía para verificación de fetos restantes y evaluación de la estática fetal. La bioquímica sérica reveló hipo calcemia moderada y el tratamiento se llevó a cabo con gluconato de calcio y ocitocina, ocurriendo la aparición de los fetos. La distocia en la clínica de perros y gatos es considerada una emergencia. La estabilización del animal es de suma importancia. La realización de exámenes de laboratorio deben ser recomendados para evaluar la ocurrencia de disturbios

DOI: https://doi.org/10.25110/arqvet.v18i3.2015.5539

'Doutoranda do Programa de Pós-Graduação em Medicina Veterinária da Universidade Federal de Campina Grande, campus de Patos, Paraíba. End: Rua Dr. José Genuíno, 361, apt. 303, Centro, Patos, Paraíba, Brasil. E-mail: nandinhavh@gmail.com.

${ }^{2}$ Médica Veterinária, Autônoma, Clínica Veterinária Amigo Sadio. End: Rua Avenida Presidente João Pessoa cep: 58220000, Bananeiras, João Pessoa, Paraíba. Email: angelmix@hotmail.com

${ }^{3}$ Médica Veterinária, Autônoma, Climev Veterinários. End: Rua Presidente Costa e Silva, 593 apt. 303, Santa Rosa - Campina Grande. Email: amandaangelocca@gmail.com

${ }^{4}$ Médica Veterinária, Mestre, Hospital Veterinário da UFCG. End: Rua 5 de agosto, 173, apto 201, bl A, Novo Horizonte, Patos, Paraíba, Brasil. E-mail: rosileide_carneiro@yahoo.com.

${ }^{5}$ Médica Veterinária, Doutora, Professora Adjunta da Universidade Federal de Campina Grande, campus de Patos, Paraíba. End: Avenida Universitária S/N

- Bairro Santa Cecília - Cx Postal 61 - Patos/PB, CEP: 58708-110. Email: nlucia@cstr.ufcg.edu.br 
electrolíticos, promoviendo un tratamiento adecuado y mejorando el pronóstico del paciente.

PALABRAS CLAVE: Parto distócico. Hipo calcemia. Hipoglucemia.

\section{Introdução}

Distocia é definida como a incapacidade de expulsão do feto por meio do canal do parto durante a parição (MONTENEGRO, 2010), apresentando ocorrência incomum em pequenos animais, com índices que variam entre cinco a seis porcentos em cadelas e gatas (NELSON; COUTO, 2010), podendo chegar até $100 \%$ em determinadas raças (FERRO, 2012).

A distocia pode ter origem materna, fetal ou de ambos (REICHLER; MICHEL, 2009; NELSON; COUTO, 2010). Segundo Ferro (2012) nas causas maternas de distocia destacam-se os distúrbios de contração, tais como, inércia uterina primária ou secundária e hipertonia uterina. A causa mais comum de distocia na cadela é a inércia uterina primária, representando, aproximadamente, $75 \%$ dos casos de distocia (JUTKOWITZ, 2005; KUTZLER, 2009).

A inércia uterina primária é definida como a ocorrência de fracas contrações sem frequência adequada, que não são eficazes na expulsão do feto por meio do canal do parto, podendo ser causada por anormalidades anatômicas ou distúrbios da interação fisiológica entre hormônios e eletrólitos (LINDE-FORSBERG, 2005). Por outro lado, a hipoglicemia em cadelas com inércia uterina primária é um achado incomum (BERGSTROM, 2009).

Para diagnosticar a distocia é necessária a realização de uma boa anamnese, detalhando todo o histórico do animal, exames físico, radiológico, ultrassonográfico, bem como hemograma e dosagens de cálcio, glicose, ureia e creatinina (LUZ et al., 2005).

A suspeita de parto distócico é sempre uma emergência, requerendo rápida avaliação clínica, podendo ser tratado por meios manipulativos, médico ou cirúrgico (LUZ et al., 2005). Segundo Jutkowitz (2005) o tratamento médico tem um prognóstico reservado, apresentando taxas de sucesso entre os 20 e os $40 \%$.

Tendo em vista que são raros os casos de cadelas em distocia com hipoglicemia (LUZ et al., 2005), este trabalho foi conduzido com a finalidade de relatar um caso de distocia em cadela associada a choque hipoglicêmico.

\section{Relato de Caso}

Um canino, fêmea, SRD, de cinco anos de idade, em segunda prenhez, pesando $14 \mathrm{~kg}$, foi atendido no Hospital Veterinário da Universidade Federal de Campina Grande, Centro de Saúde e Tecnologia Rural, Campus de Patos, Paraíba, Brasil, com o histórico de ter entrado em trabalho de parto havia, aproximadamente, quatro horas, tendo parido três filhotes mortos.

No exame clínico observou-se magreza, mucosas pálidas, hipotermia, angústia respiratória, abdome distendido, mamas crescidas com produção de leite, contrações abdominais, fetos palpáveis sem movimentação aparente, ausculta cardíaca fetal, desidratação, secreção vaginal esverdeada, obnubilação, permanecendo o animal em decúbito lateral. A glicemia foi aferida com o auxílio de glicosímetro portátil ${ }^{1}$, apresentando valores de $38 \mathrm{mg} / \mathrm{dL}$. Foram colhidas amostras de sangue para realização de exames laboratoriais (hemograma, dosagens de ureia, creatinina, cálcio, fósforo e alanina aminotransferase) e instituída terapêutica emergencial (Figura 1) mediante de fluidoterapia intravenosa com solução de Ringer com Lactato $^{2}$ adicionado de complexo polivitamínico energético ${ }^{3}$ e uma ampola de glicose $50 \%{ }^{4}$, além de administração de dexametasona ${ }^{5}$ na dose de $0,25 \mathrm{mg} / \mathrm{kg}$, por via intravenosa.

Figura 1: Cadela com distocia materna associada ao choque hipoglicêmico recebendo fluidoterapia com Ringer-Lactato e polivitamínico.

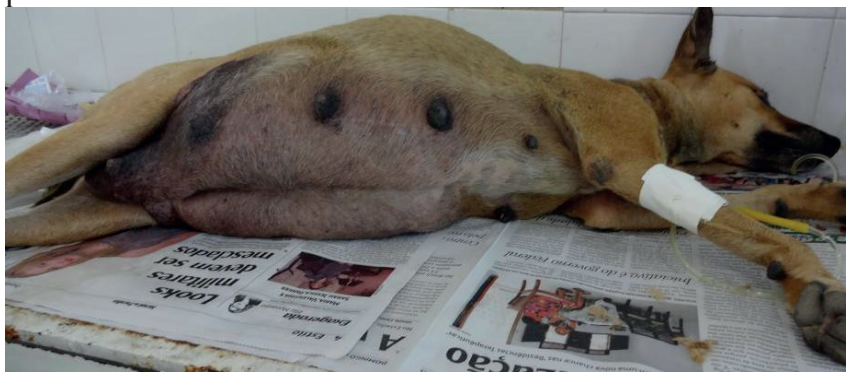

Aproximadamente 25 minutos do início da terapêutica emergencial para correção da hipoglicemia o animal recuperou-se do quadro de fraqueza e letargia e pariu um filhote (Figura 2). Neste momento a glicemia foi aferida novamente e apresentou valores de $68 \mu \mathrm{g} / \mathrm{dL}$. O neonato evolui para cianose e hipotermia. Seu exame revelou fenda palatina (Figura 2), e o mesmo veio a óbito. Decorridos 50 minutos da primeira parição, a parturiente pariu um filhote normal. Logo a seguir com a paciente consciente, ativa e com contrações abdominais foi realizada a radiografia para verificação de fetos remanescentes e avaliação da estática fetal, visualizando-se cinco fetos viáveis e em estática adequada.

Figura 2: A - Cadela com distocia materna associada ao choque hipoglicêmico ao parir o primeiro filhote após a administração de glicose a $50 \%$. B - Fenda palatina presente no filhote.

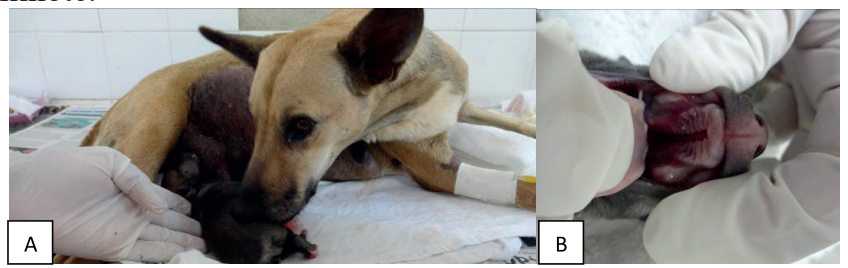

Os resultados dos exames laboratoriais demonstraram anemia normocítica normocrômica, leve linfopenia e monocitose, com hipocalcemia moderada $(6,4 \mathrm{mg} / \mathrm{dL})$.

Após aproximadamente, uma hora da parição dos

${ }^{1}$ Accu-ChekPerforma, Roche, São Paulo - SP.

${ }^{2}$ Solução de Ringer com Lactato, Equiplex Indústria Farmacêutica, Aparecida de Goiânia - GO.

${ }^{3}$ Complexo polivitamínico energético, Hertavita, Hertape Calier Saúde Animal, Juatuba - MG.

${ }^{4}$ Glicose $50 \%$, Geyer, Porto Alegre - RS

${ }^{5}$ Azium injetável 10mL, 2mg/mL, Schering Plough, São Paulo - SP. 
primeiros fetos, o parto não havia progredido e a paciente apresentava-se extremamente inquieta. Nova glicemia resultou em $259 \mu \mathrm{g} / \mathrm{dL}$ e a reposição de glicose foi suspensa. Assim, optou-se pela administração de $5 \mathrm{~mL}$ de gluconato de cálcio $^{6}$, por via intravenosa lenta, seguida pela administração de 6 UI de ocitocina ${ }^{7}$ por via intramuscular. Decorridos 40 minutos reaplicou-se a ocitocina na dose de 10 UI. A paciente foi isolada em ambiente tranquilo apenas com seus filhotes e após, aproximadamente, duas horas foi constatada a parição dos filhotes remanescentes (Figura 3).

Figura 3: Cadela com todos os filhotes após parição.

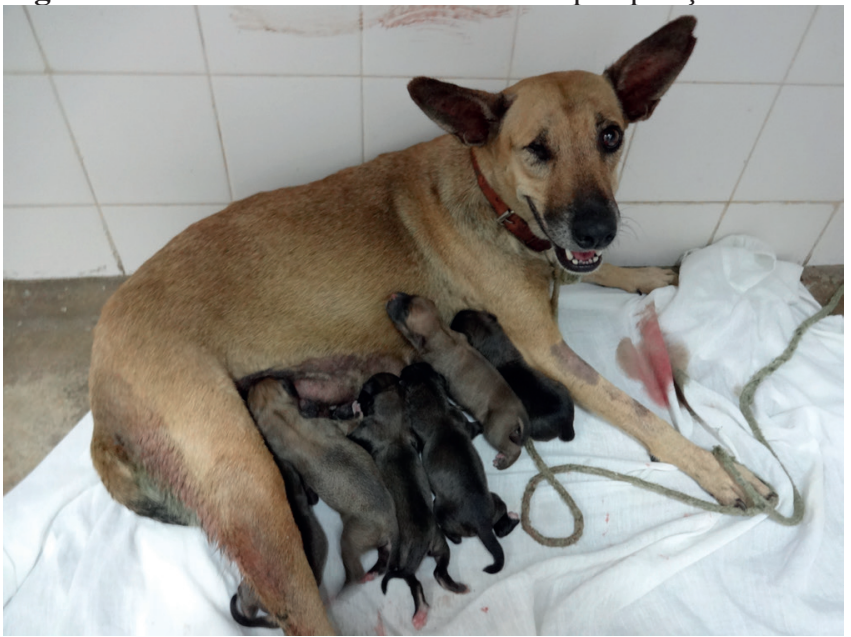

\section{Discussão}

A idade pode ser um fator relevante na incidência de distocia. Bergström et al. (2006) e Costa (2010) referem uma idade média de ocorrência desta afecção de 4,6 anos e 5,8 anos, respectivamente. Portanto, a paciente estava na média de idade predisposta para esta condição clínica.

O quadro clínico observado durante o exame clínico foi característico de distocia, concordando com Jutkowitz (2005). Além disso, foi observado corrimento vulvar esverdeado o qual é indicativo de separação placentária (SMITH-CARR, 2005).

Nos casos de distocia a realização de exames laboratoriais é necessária para avaliação do estado geral da fêmea e deve incluir a realização de hemograma e dosagens de ureia e creatinina, segundo a clínica do paciente (LUZ et al., 2005). A dosagem de cálcio e glicose pode ser solicitada quando a paciente apresentar sinais clínicos que indiquem hipocalcemia ou hipoglicemia, tais como tetanias, fraqueza, hipertermia ou até convulsões (JOHNSTON; KUSTRITZ; OLSON, 2001). Luz (2004) refere que a realização de radiografia em casos de distocia é importante para verificar a estática fetal além de prover informações sobre a quantidade de fetos. A radiografia nesses casos também pode ser realizada com o intuito de descartar anomalias ósseas do canal pélvico. Assim, pode-se dizer que a paciente foi submetida aos exames laboratoriais e de imagem adequados para o caso.

$\mathrm{Na}$ paciente deste estudo os valores séricos de glicose estavam abaixo do limite mínimo para cães que é de

${ }^{6}$ Gluconato de cálcio UCB, Agroline Produtos Agropecuários, Campo Grande - MS.

${ }^{7}$ Ocitocina HC, Hertape Calier Saúde Animal, Juatuba - MG.
65 a $110 \mathrm{mg} / \mathrm{dL}$ (BROBST, 1997). Esse quadro clínico provavelmente decorreu de subnutrição, pois, segundo Jackson (2005) há maior risco de hipoglicemia em cadelas com baixo peso corporal ao final da gestação e início da lactação. Além disso, o elevado número de fetos também pode ter contribuído para a ocorrência de hipoglicemia, pois cadelas cuja ninhada é muito grande apresentam restrição da ingestão de alimentos no final da gestação por falta de espaço estomacal (JOHNSTON; KUSTRITZ; OLSON, 2001). Para Nelson e Couto (2010) os sinais clínicos da hipoglicemia são decorrentes da neuroglicopenia e geralmente se iniciam quando a glicose está inferior a $45 \mathrm{mg} / \mathrm{dL}$.

O tratamento emergencial com ringer lactato, vitaminas e glicose buscou estabilizar o quadro geral do animal, obtendo êxito ao recuperar a paciente rapidamente. De acordo com Kutzler (2009) a hipoglicemia pode ser corrigida pela administração intravenosa de uma solução contendo glicose.

A administração de dexametasona na fêmea possibilitou a melhora da condição respiratória dos filhotes, uma vez que o aumento dos níveis de corticosteroides contribui para a produção de surfactantes, importantes na maturação pulmonar, auxiliando na respiração neonatal e no preparo para a vida extrauterina (FELDMAN; NELSON, 1996). Além disso, a administração dessas substâncias também auxilia no aumento da glicemia, à medida que aumentam a resistência à insulina diminuindo a utilização periférica de glicose e promovendo a neoglicogênese (DAMIANI et al., 2001).

Segundo Locatelli, Cury e Pereira (2009), distocias que são de causa fetal podem ser provocadas por má formação congênita. Contudo, a fenda palatina apresentada pelo primeiro feto expulso não seria capaz de gerar um quadro de distocia de origem fetal.

Os achados de hemograma observados foram os esperados nos quadros de prenhez (SMITH, 2007; VERSTEGEN-ONCLIN; VERSTEGEN, 2008), mas poderiam refletir o estado geral de debilidade do animal, bem como o estresse desencadeado no parto.

A hiperglicemia transitória verificada posteriormente pode ser atribuída a diversos fatores que incluem a administração da glicose, do glicocorticoide e ao próprio estresse desenvolvido pela parturiente ao recobrar a consciência, uma vez que, a elevação da cortisolemia devido ao estresse determina aumento da glicogênese hepática e, consequentemente, hiperglicemia (LAMONT; TRANQUILLI; GRIMM, 2000).

Hollinshead et al. (2010) sugerem que a diminuição dos níveis séricos de cálcio desempenha um papel fundamental na ocorrência de inércia uterina primária canina, completa ou parcial. Os mesmos autores afirmam que o aumento do pH e a diminuição da pressão de dióxido de carbono que ocorrem no início do parto conduzem a uma condição de alcalose respiratória subclínica que, por sua vez, resulta numa diminuição da resposta tecidual ao paratormônio. Isto leva à diminuição transitória dos níveis séricos de cálcio e à consequente diminuição da contratilidade uterina, resultando em distocia.

Embora a maioria dos estudos recomende inicialmente a administração de ocitocina seguida de gluconato de cálcio, geralmente, se o intervalo entre a expulsão de cada feto excede os 60 minutos ou se verifica inércia uterina, realiza-se primeiro a administração de gluconato de cálcio a 10\% (WIEBE; HOWARD, 2009), objetivando-se gerar au- 
mento da força de contração uterina (LUZ, 2004), conforme realizado neste caso. A administração de ocitocina, que foi repetida com o intervalo de 30 minutos, está de acordo com a literatura (JOHNSTON; KUSTRITZ; OLSON, 2001). A ausência de resposta à aplicação do gluconato de cálcio pode ter ocorrido devido ao fato de o cálcio intracelular envolvido na contração do miométrio necessitar passar para dentro da célula via canais iônicos, sendo esse transporte regulado por hormônios (SANBORN, 2000).

Pelo histórico, achados clínicos, laboratoriais e de avaliação radiológica, a distocia nesse caso pôde ser classificada como por inércia uterina primária uma vez que não havia um canal de parto obstruído, havendo associação com distúrbio eletrolítico caracterizado por hipocalcemia e hipoglicemia (MONTENEGRO, 2010). Ainda deve-se observar a influência do grande número de fetos, pois, segundo Darvelid e Linder-Forsberg (1994) a superfetação é um fator de predisposição da ocorrência de inércia uterina primária.

Em virtude da presença de contrações, ausência de anormalidades de estática fetal, e também da visualização da disposição materna após a aplicação de fármacos hiperglicemiantes, não foram indicadas manobras obstétricas manipulativas, ou ainda encaminhamento para a cirurgia de cesariana. Optar por tratamento cirúrgico é apenas uma alternativa quando a fêmea não responde ou se a resposta é fraca e demorada ao tratamento medicamentoso (LUZ, 2004), ou ainda se esta apresenta sinais de endotoxemia ou septicemia, morte fetal com putrefação ou quando há antecedentes de distocia (JOHNSTON; KUSTRITZ; OLSON, 2001).

Deve-se considerar que o animal encontrava-se estressado face ao ambiente e presenças de pessoas estranhas, motivo da aparente inquietude. Pacientes neste quadro podem prolongar o segundo estágio do parto por um tempo considerável (GAUDET; KITCHELL, 1985); essa forma, o isolamento do mesmo contribuiu para a continuação e resolução do processo de parição.

\section{Conclusão}

A distocia na clínica de cães e gatos é muito comum, sendo esta afecção considerada de emergência. A estabilização do animal é de extrema importância para a saúde, tanto da parturiente como dos fetos. A realização de exames laboratoriais deve ser preconizada no intuito de se avaliar a possível ocorrência de distúrbios eletrolíticos, tais como, a hipoglicemia e a hipocalcemia, promovendo um adequado tratamento e melhorando o prognóstico da paciente.

\section{Referências}

BERGSTRÖM, A. et al. Incidence and breed predilection for dystocia and risk factors for cesarean section in a swedish population of insured dogs. Veterinary Surgery, Philadelphia, v. 35, n. 8, p. 786-791, 2006.

BERGSTRÖM, A. Dystocia in the Bitch: Epidemiology, aetiology and treatment. Uppsala, 2009. $46 \mathrm{f}$. Tese (Doutorado). Faculdade de Medicina Veterinária e Zootecnia, Universidade Sueca de Ciências Agrícolas.

BROBST, D. F. Pancreatic function. In: KANEKO J. J.;
HARVEY, J. W.; BRUSS M. L. Clinical biochemistry of domestic animals. 5. ed. San Diego: Academic Press, 1997. p. 353-366.

COSTA, T. I. R. da. Urgências reprodutivas na cadela. Lisboa, 2010. 101 f. Dissertação (Mestrado Integrado em Medicina Veterinária) - Faculdade de Medicina Veterinária, Universidade Técnica de Lisboa Lisboa, 2010.

DAMIANI, D. et al. Corticoterapia e suas repercussões: a relação custo-benefício. Pediatria, São Paulo, n. 1, p. 7182, 2001.

DARVELID, A.W.; LINDE-FORSBERG, C. Dystocia in the bitch: a retrospective study of 182 cases. Journal of Small Animal Practice, Oxford, v. 35, n. 8, p. 402-7, 1994.

FELDMAN, E. D.; NELSON, R. W. Canine and feline endocrinology and reproduction. 2. ed. Philadelphia: W.B. Saunders, 1996. 778 p.

FERRO, A. C. Distocia na Acupuntura Veterinária. Revisão de Literatura. Campinas, 2012. 32 f. Monografia (Especialização em Acupuntura Veterinária) - Instituto Homeopático Jacqueline Peker.

\section{GONZÁleZ, F. H. D.; SILVA, S. C. Patologia Clínica}

Veterinária: Texto Introdutório. Porto Alegre:

Universidade Federal do Rio Grande do Sul, 2008. 342 p.

HOLLINSHEAD, F. K. et al. Calcium, parathyroid hormone, oxytocine and $\mathrm{pH}$ profiles in the whelping bitch. Theriogenology, Stoneham, v. 73, n. 9, p. 1276-1283, 2010.

JACKSON, P. G. G. Obstetrícia veterinária. 2. ed. São Paulo: Rocca, 2005. 328 p.

JOHNSON, C. A. Pregnancy management in the bitch. Theriogenology, Stoneham, v. 70, n. 9, p. 1412-1417, 2008.

JOHNSTON, S. D.; KUSTRITZ, M. V. R.; OLSON, P. N. $\mathrm{S}$. Canine and Feline Theriogenology. 1. ed. Filadelfia: Saunders, 2001. 592 p.

JUTKOWITZ, L. A. Reproductive emergencies. Veterinary Clinics of North America: Small Animal Practice, Philadelphia, v. 35, n. 2, p. 397-420, 2005.

KUSTRITZ, M. V. R. Clinical canine and feline reproduction: evidence-based answers. Iowa: Blackwell Publishing, 2010. 316 p.

KUTZLER, M. A. Dystocia and obstetric crises. In: SILVERSTEIN, D. C.; HOPPER, K. Small animal critical care medicine. St. Louis, Missouri: Elsevier Saunders, 2009. p. 611-615.

LAMONT, L. A.; TRANQUILLI, W. J.; GRIMM, K. A. Physiology of pain. Veterinary Clinician of North American Small Animal Practice, v. 30, n. 4, p.703-728, 2000 . 
LINDE-FORSBERG, C. Abnormalities in pregnancy, parturition, and the periparturient period. In: ETTINGER, S. J. et al. Textbook of Veterinary Internal Medicine. Philadelphia: W.B. Saunders, 2005. p. 1655-1667.

LOCATELLI, L.; CURY, J. R. L. M.; PEREIRA, D. M. Estática Fetal. Revista Científica Eletrônica de Medicina Veterinária, Garça, Ano VII, n. 12, 2009.

LUZ, M. R. Parto en perras y gatas. In: GOBELLO, C. Temas de reproducción de caninos y felinos por autores latinoamericanos. La Plata: Gráfica Latina, 2004. p. 237248.

LUZ, M. R. et al. Gestação e parto em cadelas: fisiologia, diagnóstico de gestação e tratamento das distocias. Revista Brasileira de Reprodução Animal, Belo Horizonte, v. 29, n. 3/4, p.142-150, 2005.

MONTENEGRO, L. M. F. Estudo retrospectivo de urgências reprodutivas no Hospital Veterinário Montenegro. Villa Real, 2010. 63 f. Dissertação (Mestrado em Medicina Veterinária) - Escola de Ciências Agrárias e Veterinárias, Departamento de Ciências Agrárias, Universidade de Trás-os-Montes e Alto Douro.

NELSON, R. W.; COUTO, C. G. Medicina Interna de Pequenos Animais. Rio de Janeiro: Elsevier, 2010. 1674 p.

REICHLER, I. M.; MICHEL, E. Dystocia: recognition and management. European Journal of Companion Animal Practice, Zurique, v. 19, n. 2, p. 165-173, 2009.

SANBORN, B. M. Relationship of ion channel activity to control of myometrial calcium. Journal of the Society for Gynecologic Investigation, New York, v. 7, n. 1, p. 4-11, 2000

SMITH-CARR, S. Gynecologic emergencies. In: ETTINGER, S. J.; FELDMAN, E. C. Textbook of veterinary internal medicine: diseases of the dog and cat. 6. ed. St. Louis, Missouri: Elsevier Saunders, 2005. p. 450-452.

SMITH, F. O. Challenges in small animal parturition - timing elective and emergency cesarian sections.

Theriogenology, Stoneham, v. 68, n. 3, p. 348-353, 2007.

VERSTEGEN-ONCLIN, K.; VERSTEGEN, J.

Endocrinology of pregnancy in the dog: a review.

Theriogenology, Stoneham, v. 70, n. 3, p. 291-9, 2008.

WIEBE, V. J.; HOWARD, J. P. Pharmacologic advances in canine and feline reproduction. Topics in Companion Animal Medicine, New York, v. 24, n. 2, p. 71-99, 2009. 